\title{
Analyses of a polyphenol aglycone profile in broccoli and carrots by LC-MS QToF
}

\author{
L. L. Hamill ${ }^{1}$, W. C. McRoberts ${ }^{2}$, S. D. Floyd ${ }^{2}$, M. C. McKinley ${ }^{1}$, I. S. Young ${ }^{1}$ and J. V. Woodside ${ }^{1}$ \\ ${ }^{1}$ Nutrition \& Metabolism Group, Centre for Public Health, Queen's University Belfast BT12 6BJ, UK and \\ ${ }^{2}$ Food Chemistry Division, Agri-Food and Biosciences Institute, Newforge Lane, Belfast BT9 5PX, UK
}

Epidemiological data indicates that increased intake of fruit and vegetables (FV) is associated with decreased incidence of chronic diseases ${ }^{(1-2)}$. FV contain an array of potentially protective components which may account for the health benefits associated with increased intakes. This array includes polyphenols, which are predominantly flavonoids ${ }^{(3)}$. However, to determine polyphenol intake from FV it is important to accurately assess food content, and the effects of cooking. Unpublished data, collected within our research centre, identified broccoli and carrots as two of the most commonly consumed vegetables in a Northern Irish population. The aim of this study was to measure the polyphenol content of these two vegetables and to establish if domestic cooking had an effect on content. Although there are numerous published methods for analyses of individual polyphenols employing modern techniques such as liquid chromatography-mass spectrometry (LC-MS), this work utilises a sensitive and specific LC-MS method which analyses a representative panel of polyphenols within a single run. The methodology used is summarised below.

Broccoli and carrots were purchased from a local supermarket and prepared on the day of purchase. They were cooked by boiling in water for $15 \mathrm{~min}$. A raw sample $(n 2)$ and a cooked sample $(n 2)$ of each were frozen, lyophilised, milled and $0.05 \mathrm{~g}$ was hydrolysed with beta-glucuronidase and sulfatase from Helix pomatia. A profile of 14 polyphenol aglycones (including at least one representative from each of the main flavonoid subclasses and selected phenolic acids) were analysed by LC-MS quadrupole time of flight (QToF). Flavonols (kaempferol, quercetin) were detected in broccoli. Phenolic acids (para-coumaric acid, caffeic acid) were detected in broccoli and carrots.

\begin{tabular}{|c|c|c|c|c|c|c|c|c|c|c|}
\hline $\begin{array}{l}\text { Polyphenol content } \\
(\mathrm{mg} / \mathrm{kgFW})\end{array}$ & $\begin{array}{c}\text { Raw } \\
\text { broccoli }\end{array}$ & $\mathrm{SE}$ & $\begin{array}{l}\text { Cooked } \\
\text { broccoli }\end{array}$ & $\mathrm{SE}$ & $P$-value & $\begin{array}{l}\text { Raw } \\
\text { carrot }\end{array}$ & $\mathrm{SE}$ & $\begin{array}{l}\text { Cooked } \\
\text { carrot }\end{array}$ & SE & $P$-value \\
\hline Kaempferol & 19.37 & 1.34 & 16.28 & 0.19 & 0.150 & $n d$ & & $n d$ & & \\
\hline Quercetin & 2.74 & 0.17 & 5.67 & 0.22 & $<0.01$ & $n d$ & & $n d$ & & \\
\hline para-Coumaric acid & 2.15 & 0.09 & 1.34 & 0.00 & $<0.05$ & 0.73 & 0.02 & 0.85 & 0.01 & $<0.05$ \\
\hline Caffeic acid & 0.97 & 0.15 & 6.07 & 0.05 & $<0.05$ & 2.41 & 0.46 & 18.67 & 0.03 & $<0.05$ \\
\hline
\end{tabular}

$n d$, not detected; $P$-value for 'difference between group' effect, obtained by a 2-tailed independent samples t-test.

Our results indicate that polyphenols are present in these two vegetables and interestingly, cooked broccoli contained significantly higher levels of quercetin and caffeic acid than raw broccoli while cooked carrot contained significantly higher levels of para-coumaric acid and caffeic acid than raw carrot. Polyphenols in these vegetables may play a role in the beneficial effects associated with increased consumption. However, before we can fully establish the impact of polyphenols on human health, further research is required, measuring polyphenol content in other FV. The many factors which influence polyphenol content in FV, including cultivar, ripeness, location and season of growth, processing methods and preservation techniques, in addition to domestic cooking as highlighted in this abstract, also need to be explored.

This work has been funded by the Medical Research Council (MRC).

1. Dauchet L, Amouyel P, Hercberg S et al. (2006) J Nutr 136, 2588-2593.

2. Vainio H \& Weinerpass E (2006) Nutr Cancer 54, 111-142.

3. Knekt P, Kumpulainen J, Jarvinen R et al. (2002) Am J Clin Nutr 76, 560-568. 